\title{
La predicción en Economía: Posibilidades y limitaciones
}

\author{
ANTONIO PULIDo SAN RomÁn \\ Instituto L.R. Klein. Universidad Autónoma de Madrid, c/ Francisco Tomás y Valiente , 5, 28049 \\ Madrid, España.E-mail: antonio.pulido@uam.es
}

\begin{abstract}
RESUMEN
Se parte de una reflexión sobre el largo camino que separa a las ciencias en que predominan leyes de estricto cumplimiento, de aquellas otras, como la Economía, en que sólo se cuenta con regularidades de pasado y deben arriesgarse con predicciones, inciertas, de futuro. Si, además, el entorno de predicción es VUCA (volatility, uncertainty, complexity y ambiguity) la tarea se complica. Es necesario hacer una revisión crítica del pasado de la predicción económica y empresarial para pasar a una reflexión sobre su futuro.
\end{abstract}

Palabras clave: Predicción económica, modelos econométricos, escenarios alternativos.

\section{Prediction in Economics: Possibilities and Limitations}

\begin{abstract}
This article starts from a reflection on the long path that separates the sciences in which laws of strict compliance predominate, of those others, like Economy, in which only regularities of the past exist and they must risk with uncertain predictions about future. In addition, if the prediction environment is VUCA (volatility, uncertainty, complexity and ambiguity), the task becomes complicated. It is necessary to make a critical review of the past of economic and business prediction to move on to a reflection of its future.
\end{abstract}

Keyword: Economic Prediction, Econometric Model, Alternative Scenarios.

Clasificación JEL: C53, C50

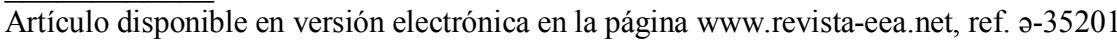




\section{DE LEYES DE ESTRICTO CUMPLIMIENTO A REGULARIDADES DE PASADO Y PREDICCIONES DE FUTURO}

Valorar el pasado, presente y futuro de la predicción en el amplio campo de la Economía y la Empresa, exige empezar por unas reflexiones básicas sobre el papel de la predicción en la ciencia en general. Porque en todos los campos científicos existe una actividad predictiva, pero con características muy diferentes. Predicen el físico, el ingeniero, el meteorólogo, el médico, el sociólogo o el economista.

La gran diferencia está en que en las áreas experimentales predominan unas leyes para un entorno estable, que permiten pronosticar con elevada certeza, incluso con exactitud, los resultados de una actuación. Cuando los entornos son más variables, como en medicina o meteorología, en los resultados empiezan a predominar la incertidumbre, aunque existan leyes válidas para circunstancias estándar.

En el campo económico y en las ciencias sociales en general, las leyes de obligado cumplimiento se refieren a situaciones teóricas simplificadas. En la práctica, cada situación es un caso nuevo, en que cambian rápidamente estructuras, decisiones de los agentes y entornos socio-políticos.

Hace medio siglo, Fox, Sengupta y Thorbecke (1966) describían la situación diferencial entre científicos con unas frases que me siguen pareciendo relevantes: Los científicos e ingenieros que diseñan vehículos espaciales están en una posición envidiable. Comienzan con teorías que un lego encontraría inalcanzables y aplican transformaciones matemáticas que encontraría incomprensibles. Pero al final colocan en órbita un vehículo que brilla como una estrella; aparece en el cielo de la noche cuando y donde ellos dijeron; y su éxito es visiblemente evidente no solo para el propio lego, sino para su hijo de cinco años. El economista que crea una nueva teoría con la ayuda de matemática avanzada es respetado por sus propios colegas. Pero el lego permanece muy estirado, impasible a las prescripciones politicas derivadas de ella: "puede ser correcto en teoría pero no funcionará en la práctica".

Podremos, los economistas, aplicar técnicas estadístico-econométricas más o menos complejas y depuradas; dominar las teorías y leyes económicas más elaboradas y actuales; crear y depurar los datos más completos y recientes. Al final la predicción que realicemos será una actividad de alto riesgo, a la que algunos, incluso, niegan un carácter científico.

En particular, los seguidores de la Escuela Austriaca de Economía (Menger, Hayek, Mises,...) consideran que no hay posibilidad de un conocimiento científico (objetivo) del futuro, limitándose la predicción económica a un conocimiento práctico (subjetivo): "Corresponde, pues, al hombre en general y 
al empresario en particular realizar predicciones sobre la evolución futura de los acontecimientos, utilizando para ello sus conocimientos teóricos y su experiencia. Pero el científico de la Economía de ninguna manera, como tal científico, puede efectuar predicción concreta alguna, es decir de naturaleza cuantitativa, geográfica y temporal determinada" (J. Huerta de Soto, 2004, pág. 60). Más aún: "la información que proporciona la experiencia histórica no puede utilizarse para construir teorias y predecir eventos futuros" y "la Econometría no es más que un puro juego de niños que en nada contribuye a elucidar los problemas económicos de la realidad" (pág. 64 y 66).

No es preciso llegar a estos radicalismos metodológicos, ni aceptar el irónico comentario de la cita de John Kennet Galbraith de que "la única función de la predicción económica es hacer que la astrología parezca respetable", para reconocer las limitaciones de las predicciones económicas en general y de los modelos econométricos en particular o remontarnos a las discrepancias teóricas entre escuelas de pensamiento.

En mi blog escribí hace unos meses (blog.antoniopulido.es/por-que-loseconomistas-no-se-ponen-de-acuerdo) unas consideraciones generales que creo definen mi posición.

Hay múltiples razones para que los economistas (profesionales o aficionados) tengan opiniones diferentes sobre los temas más variados de su campo. A veces son sesgos, interesados o no, de carácter ideológico. En ocasiones se trata de experiencias, información y conocimientos diversos. Pero, en el fondo, es la consecuencia de una ciencia social que trabaja en el complejo entramado de las decisiones de individuos o instituciones, donde no hay leyes fijas de comportamiento, sino respuestas a las circunstancias múltiples (económicas, sociopolíticas, tecnológicas,...) de un mundo en continuo cambio.

Pero una cosa es que no haya leyes fijas e inmutables y otra es que sea un territorio arbitrario sin leyes. En las ciencias más duras, como la física, hay leyes que se cumplen siempre (al menos dentro de unos límites) y no hay discusión sobre las mismas. En otras ciencias menos deterministas, como las vinculadas al campo de la medicina, al menos se cuenta con que los organismos del cuerpo humano son muy similares entre individuos y no cambian, en lo fundamental, con el tiempo $¡ Y$, a pesar de ello, los médicos tienen discrepancias en diagnósticos y terapias!

¿Qué cabe esperar entonces en Economía, donde la situación es como si las leyes que regulan el funcionamiento del corazón o el hígado fuera diferente para cada individuo y además estuvieran en permanente proceso de cambio, condicionado por múltiples causas externas? A pesar de estas dificultades, la ciencia económica ha avanzado hasta disponer de un extenso y especializado campo con leyes teóricas y empíricas. Eso sí, al coste de diagnósticos y tratamientos cambiantes por escuelas de pensamiento e incluso a escala 
individual.

Pero con la múltiple y creciente información económica ¿no se pueden establecer leyes empíricas válidas para un país o una empresa en un momento dado? Sobre esta cuestión hay dos posiciones extremas: la de quienes tienen una confianza plena en que los datos permiten validar o rechazar teorías y concretar, en cada caso, esas leyes; y la de los que niegan incluso la posibilidad de establecer relaciones numéricas entre variables, al menos con carácter general y como herramienta útil de ayuda a la toma de decisiones económicas.

Debo hacer, previamente, una referencia a los "modelos econométricos", como la forma más habitual y evolucionada de establecer relaciones empíricas entre variables. En el caso más simple, si partimos de una relación causal entre p. ej. el consumo de los hogares y la renta disponible, podemos cuantificar una relación funcional a partir de datos de diferentes años para un país.

Pongamos ahora juntas múltiples variables en diversas ecuaciones para explicar el crecimiento de una economía, su nivel de paro o de endeudamiento y tendremos una especie de "maqueta numérica simplificada". Si conseguimos una aproximación suficiente al funcionamiento de la realidad, disponemos de una herramienta que permite comprobar la veracidad de supuestos teóricos, valorar la efectividad de políticas económicas alternativas o predecir evoluciones a futuro, al menos si no hay grandes cambios de funcionamiento respecto a la realidad observada.

Admitir esta situación óptima lleva a una posición metodológica a la que he denominado en algunos de mis libros como "La Gran Ingenuidad". Existen muchos motivos para que falle la "maqueta" a la hora de explicar una realidad económica, aunque aquí sólo me referiré a algunos de los más relevantes:

El primero es la relativa arbitrariedad a la hora de seleccionar las variables del modelo. La ideología, la experiencia y los recursos del modelizador, condicionan la selección inicial de variables. Y si no se introducen ciertas variables, no sólo no saldrán nunca como relevantes, sino que dejarán hueco para que ganen peso otras que pueden ser más secundarias.

El segundo motivo es la limitación de información disponible, las alternativas de cómputo y la discrepancia entre fuentes de datos. Si no disponemos de información de alguna variable, o la descartamos o la estimamos indirectamente a través de indicadores o "proxys".

El tercero es la posibilidad de utilizar periodos temporales más o menos amplios o seleccionar países de comparación (datos trasversales). Naturalmente cabe la posibilidad de que la selección sea interesada para llegar a ciertos resultados pero, sobre todo, evidencia que incluso con un mismo modelo de partida puede llegarse a resultados diferentes.

Aún cabe añadir las alternativas técnicas disponibles a la hora de "estimar" 
los coeficientes del modelo o de introducir "factores de ajuste". En resumen, es ingenuo aceptar que un modelo econométrico tiene la garantía de proporcionar una visión objetiva y sólidamente fundada del funcionamiento empírico de la economía de un país, sector o empresa.

Frente a la gran ingenuidad extrema está la posición radical que niega el pan y la sal a todo ejercicio de cuantificación, con argumentos (de validez sólo parcial) sobre olvido de lo cualitativo en una ciencia humana, definición instrumentalista de conceptos, correlaciones espurias tomadas como causalidades u otras múltiples limitaciones que muchos reconocemos de partida, pero que no permiten renunciar a una herramienta que tiene gran valor, siempre utilizada con prudencia.

Hay un campo de aplicación de los modelos que también se encuentra entre los polos extremos de ingenuidad crónica y de negación radical: la predicción económica y empresarial.

Partamos del reconocimiento de que hay muy diversas formas de predicción y que los modelos econométricos sólo son una de las opciones disponibles. Más aún, mi experiencia es que la mejor predicción se obtiene utilizando enfoques y herramientas múltiples, que no es cuestión de detallar aquí.

Admitamos también que predecir el futuro no es una opción sino un ejercicio imprescindible para cualquier tomador de decisiones económicas, sea a nivel personal, empresarial o macro. Lo que decidimos hoy tiene consecuencias en el mañana y sería erróneo hacerlo a partir de lo que está ocurriendo o de nuestra experiencia más reciente.

Debemos aceptar que existe una dificultad radical para conocer un futuro que no está escrito y que depende de nuestras propias decisiones y de un entorno incierto y cambiante. Debemos reconocer que los datos del pasado no son suficientes para obtener predicciones del futuro, tanto más cuanto más lejano es el horizonte de predicción. Tenemos que admitir que hay frecuentes errores de predicción y correcciones continuas.

Pero como en meteorología o en otras ciencias sociales es obligación de los economistas internarse en el peligroso, intrincado y cambiante campo del futuro, intentando superar limitaciones, con una visión no puramente economicista y más allá de la parcialidad de enfoques y técnicas. La predicción no es un resultado de una técnica. No es la pura extrapolación a futuro de leyes del pasado. Es una apuesta necesariamente limitada y arriesgada de futuro, que debe someterse a una revisión permanente y tener en consideración todo tipo de condicionantes, económicos y extraeconómicos.

En resumen, los economistas debemos ser conscientes de nuestras limitaciones a la hora de explicar o predecir los fenómenos que se producen en nuestro campo, pero también de que disponemos de herramientas cuantitativas que hacen nuestra tarea más efectiva. 


\section{JUICIO A LA PREDICCIÓN}

Creo haber mostrado en libros, artículos académicos, post en mis blogs y comentarios en redes sociales, mi escepticismo sobre la exactitud de las predicciones económicas. Los que nos dedicamos profesionalmente a esta arriesgada tarea, sabemos bien las múltiples razones que hay para errar cuando queremos adentrarnos en el futuro.

La primera y principal es que en economía (y en las ciencias sociales más en general) ese futuro que tratamos de pronosticar depende de las decisiones que tomen políticos, empresarios o ciudadanos del mundo entero, sin olvidar guerras, ataques terroristas o catástrofes naturales.

Intentar conocer el futuro es, en principio, un acto de soberbia, sólo disculpable por la imperiosa necesidad que todos tenemos de tomar decisiones en circunstancias que afectaran a nuestras vidas durante meses o años. No apostar por un futuro, aunque sea corriendo riesgos, equivale a actuar como si la situación actual fuera fija o, al menos, extrapolable en el tiempo. Pero los grandes riesgos y oportunidades se producen, precisamente, por los cambios profundos que suceden en el contexto económico y social en que todos nos movemos.

Pero, ¿qué utilidad pueden tener apuestas de futuro que son imprecisas, muchas veces erróneas y además se revisan permanentemente? La respuesta es que carecerían de utilidad si los predictores no disponen de herramientas de análisis e información diferencial respecto a los tomadores de decisión o si estos no son capaces de adaptarse los inevitables cambios de situación.

La realidad es que los centros de predicción profesionales (raramente es una actividad individual, al menos a nivel macro, dada la complejidad de la tarea) trabajan con variados medios y datos, que van desde sofisticados modelos econométricos, a indicadores, encuestas o información subjetiva. Como hay diferentes procedimientos, experiencias y habilidades, los aciertos y errores se distribuyen entre centros y existen paneles que incluyen predicciones comparativas y consensos (habitualmente simples promedios).

Un análisis objetivo de la fiabilidad de las predicciones macroeconómicas, pone de manifiesto la existencia de grandes fallos, sobre todo en periodos de crisis. Posiblemente los economistas metidos en labores de expectativas de futuro, debiéramos incluir explícitamente en la receta los riesgos y contraindicaciones posibles del producto que suministramos. Porque conocer, de verdad, lo que va a terminar ocurriendo en temas económicos es una entelequia, sobre todo cuando cambian las tendencias y estructuras del pasado, es decir cuando más se necesita.

Pasemos, sobre estas bases, al tema concreto de si ha habido predicciones que avisaran de la llegada de la pasada crisis. Una recesión como la ocurrida, principalmente, en el periodo 2008-2013, no ha sido prevista en forma cuantitativa aproximada antes de su inicio. Se han adelantado posibles impactos 
del estallido de la burbuja inmobiliaria y de reestructuración financiera, que se trasladaron, tímidamente, a una desaceleración progresiva del crecimiento. Pero nadie "imaginaba" la realidad de un "cisne negro" con quiebras en cadena e infección internacional de derivados financieros fraudulentos. Los economistas no disponemos de bola de cristal y nos tenemos que conformar con teorías, bases de datos, modelos y experiencias acumuladas, aparte de intuiciones siempre peligrosas.

Utilizando datos del informe que, mensualmente realiza Consensus Forecast con predicciones "revisadas" por países y centros de predicción, dos economistas del FMI, Hiles Ahir y Prakash Loungani publicaron un documento muy crítico sobre la habilidad demostrada para avisar del inicio y cambios de tendencia en el periodo de crisis ("There will be growth in the spring: How well do economist predict turning points", 14/4/15).

Según los datos de países analizados, se produjeron 88 caídas/pais en recesión (entendidas como tasas anualizadas negativas de variación del PIB real) durante el periodo 2008-2012 de las que sólo 11 fueron adelantadas en septiembre del año anterior y ninguna en los dos primeros años, 2008-09.

Además, añaden Ahir y Loungani, las predicciones revisadas mes a mes, muestran una corrección a la baja en los años de recesión, pero a un ritmo tan lento como para no llegar a comprometerse en un cambio drástico de tendencia. Tratando de buscar explicación a estos fallos, sugieren tres posibles explicaciones:

1. Los centros de predicción no disponen de suficiente información como para calibrar la caída en recesión, por carencias de sus modelos o el impacto de shocks inesperados.

2. Los predictores carecen de estímulos (reducido beneficio personal frente a alto coste de reputación o incluso riesgo de perder empleo en caso de fallo) como para arriesgarse a adelantar un giro de la economía hacia la recesión.

3. Aplican una táctica conservadora de revisión moderada y permanente en respuesta a la nueva información entrante.

Estoy, en grandes líneas, de acuerdo con el diagnóstico de Ahir y Loungani, al que creo necesario añadir algunos matices relevantes.

El primero es partir del erróneo supuesto de que los predictores de temas económicos disponemos de modelos que, si fueran correctos en su estructura e hipótesis de entorno, darían automáticamente el diagnóstico correcto sobre evolución futura de una economía. Eso creía yo, un joven económetra con fe de carbonero en datos y modelos, hace treinta años. La realidad es que una gran mayoría de los centros de predicción de todos los países del mundo, no utilizan modelos empíricos formalizados. Los que sí disponemos de uno o varios modelos (p.ej. para datos anuales, trimestrales, de baja frecuencia o enfoques 
macro, meso y micro) sabemos que proporcionan una valiosa congruencia interna de sus soluciones numéricas, pero también que pueden dar múltiples predicciones no siempre coincidentes.

Los resultados cambian no sólo con la estructura del modelo, sino con los datos (cambiantes y revisados de Contabilidad Nacional u otras fuentes) y con los supuestos sobre las variables de entorno del modelo (p.ej. crecimiento de los principales países del mundo, tipos de cambio, precios del petróleo, política monetaria o fiscal de la UE,...). Pero un modelo responde a una estructura, incorporada a los coeficientes de sus ecuaciones, deducida del pasado con la temeraria tarea de vislumbrar el futuro. Luego el predictor-modelizador no cuenta con un instrumento de diagnóstico único y fiable, sino con un simulador de alternativas que puede (y debe) utilizar como una herramienta más para establecer su arriesgada apuesta de futuro. Apuesta que no es sólo de crecimiento del PIB, sino de decenas o cientos de variables en los casos más elaborados.

La consideración de la actividad predictora como una mezcla de ciencia y arte, responde a un enfoque interesante, pero que exige múltiples matices y aclaraciones. Su carácter científico creo que está sólidamente sostenido por las rigurosas herramientas estadístico-matemáticas o estrictamente econométricas que utiliza. Pero es cierto que la mayoría de las predicciones económicoempresariales tienen su propia "cocina" y no son un producto puro, objetivo y limpio de toda manipulación, que muchos piensan y los más "puristas" defienden. Como ese proceso admite muchas variantes y, en último término, puede apelar a la intuición y subjetivismo del predictor, hay quienes apuntan a la existencia de un cierto "arte" en ese afán de vislumbrar el futuro.

Acepto que tanto la predicción económica, como el diagnóstico médico, la construcción de edificios o la gestión de empresas, tienen un cierto componente extra-científico que va más allá de los resultados técnicos de modelos econométricos, pruebas diagnósticas, estudio de resistencias o normas básicas de dirección y liderazgo. Esos grados de libertad que tenemos en economía, medicina, arquitectura... y en las ciencias sociales en general, puede calificarse de arte (como forma de actuar, al referirnos p.ej. al "arte de la pesca") o, simplemente, de habilidades desarrolladas por acumulación de experiencias.

Como cualquier habilidad, existen en la predicción comportamientos que pueden mejorar sus resultados y convertirse en guías recomendables, más allá de los debates puramente científicos. En ese sentido Tetlock y Gardner (2015) insisten en que podemos aprender a predecir mejor acontecimientos futuros: "está demostrado que son mejores los predictores que reflexionan más profundamente a la hora de hacer sus valoraciones y que reciben precisas y rápidas respuestas sobre el grado de error de sus juicios".

Pero el ser cuidadoso en el proceso y aprender de los errores es un consejo 
demasiado general. Personalmente creo que, en predicción económica y empresarial, hay que matizar mucho el proceso a seguir según diferentes situaciones y, muy en particular, según el horizonte temporal. Los pronósticos a corto plazo tienen mucho de ciencia y relativamente poco de arte; el futuro está fuertemente condicionado por el pasado y sólo informaciones privilegiadas de entorno pueden aportar mejoras relevantes sobre los resultados de las ya muy refinadas técnicas de proyección de series temporales y modelos econométricos estructurales.

La situación cambia rápidamente según se amplía el plazo para el que se realiza la predicción. La información y las reglas de comportamiento del pasado no son ya una herramienta segura para definir un futuro incierto a tres, cinco, diez o veinte años vista. La habilidad del predictor (el "arte", si así se quiere etiquetar) juega un papel creciente. Ya no hay una predicción técnica, sino múltiples apuestas de futuro posibles, condicionadas por los supuestos sobre un entorno cambiante.

En estas circunstancias, una predicción que no haga explícitos los supuestos sobre los que se elabora, carece prácticamente de valor informativo para el posible usuario. Se reduce a una opinión a tener más o menos en cuenta según el nivel de confianza que otorguemos al emisario.

Por ello, la utilización de escenarios o entornos de futuro alternativos es una solución recomendable, especialmente en momentos de alto riesgo y para horizontes de predicción de medio y largo plazo. La habilidad del predictor consiste en seleccionar los factores de riesgo más relevantes, valorar cuantitativamente sus alternativas de evolución y aplicar un procedimiento adecuado de análisis de impactos. No garantiza la certeza de los futuros alternativos diseñados, pero ayuda al proceso de toma de decisiones de los usuarios, en particular si se explicitan con detalle los supuestos y se asigna una probabilidad (necesariamente subjetiva) de ocurrencia a cada escenario.

Pero no olvidar: no se trata de predicciones técnicas objetivas; sólo son apuestas de futuro con una importante componente científica y una nada despreciable componente subjetiva. Son sólo incursiones útiles en el futuro que pueden ayudar a tomar decisiones en un ambiente de incertidumbre.

\section{LA PREDICCIÓN ECONÓMICA EN ENTORNOS VUCA}

En los dos apartados precedentes hemos revisado las especiales dificultades de la predicción económica. Ahora trataremos de profundizar en los cambios de enfoque que se están produciendo en los últimos años y en sus perspectivas de futuro.

En mi opinión, la gran transformación es pasar de una visión en que el pasado, los datos, constituyen la clave del futuro, a aceptar un protagonismo 
compartido de experiencias observables y de transformaciones por venir en un entorno volátil, incierto, complejo y ambiguo (VICA o VUCA por siglas en inglés).

Porque observar el presente es como mirarse en el espejo: se ve lo que eres pero no da pistas de lo que serás. Fijarse en el pasado es como mirar por el retrovisor del coche: ves lo que se te viene encima pero te puede distraer de a dónde te diriges. Para adentrarte en el mañana es necesario pasar al otro lado del espejo; dejar de ensimismarte en tu presente o en tu pasado y atreverte a entrar en lo desconocido, con todos los riesgos e incertidumbres que conlleva. Se trata de ir incluso más allá de predecir lo que parece va a ocurrir, para inventar un futuro deseado y trazar el camino para que pueda hacerse realidad.

El eslogan utilizado por Wharton, la prestigiosa Facultad de Empresariales de la Universidad de Pennsylvania, para su escuela de ejecutivos es: "Ahora invento en lugar de predecir. Soy un visionario". No se trata de renunciar a predecir el futuro, sino de ir un poco más allá, hasta imaginar un futuro que no tiene por qué ser el que parece venírsenos encima nos guste o no.

En el fondo es otra forma de pedir a quien se interna en el intrincado campo de la economía, como hizo Keynes en su momento, que "debe ser matemático, historiador, estadista y filósofo hasta cierto punto (...) para estudiar el presente en función del pasado y pensando en el futuro". Pasado, presente y futuro son partes fundamentales del análisis y la toma de decisiones en la gestión económica y empresarial. Lo difícil es encontrar el adecuado equilibrio en cada ocasión.

Un buceador en las oscuras y peligrosas aguas del futuro sin referencias adecuadas de dónde está y de cuáles son las corrientes predominantes, puede fácilmente perderse. Ser un visionario exige saber por dónde está uno moviéndose; si no puede caerse en ejercicios puramente imaginativos de reducido interés práctico.

En el extremo contrario están aquellos que aún tratan de vislumbrar el futuro como una proyección estable del pasado. Prácticamente nadie cae en la ingenuidad de pensar que el mañana será igual que el hoy o el ayer, pero sí es todavía habitual trasladar a futuro las tendencias más inmediatas.

En temas económicos, extrapolar la evolución pasada, sobre todo a medio y largo plazo, es una simplificación que conduce a altos costes en la gestión personal, empresarial o pública. Un tipo de interés al alza, un precio del petróleo a la baja, un mercado en expansión o un producto con demanda creciente, no son siempre posibles mantenerlos en el tiempo. Una predicción continuista es un ejercicio numérico limitado de escaso valor añadido, por muy complejas que sean las técnicas estadístico-econométricas que se utilicen.

Pero incluso las leyes del pasado, las "estructuras" de comportamiento, 
cambian permanentemente. Hace años, en unas conferencias que daba en la Universidad de Rosario, Argentina, algunos de los profesores asistentes, que habían seguido un posgrado en EEUU, me confesaron su decepción con los modelos empíricos que se construyen con series temporales y reproducen, por ello, a futuro comportamientos del pasado. Me decían: "Los modelos econométricos son conservadores por naturaleza y no valen en economías cambiantes y desequilibradas como las latinoamericanas, en que lo que ya sabemos es que nuestra situación y trayectoria son inaceptables a futuro".

No es cierto que todos los modelos y técnicas de predicción tengan que reproducir a futuro estructuras de pasado, pero sí lo es que esta forma de proceder es habitual. Y además la crítica no vale sólo para economías emergentes en un mundo en cambio continuo y acelerado.

Personalmente no tengo una respuesta concreta sobre cómo responder a los retos de futuro. Pero sí tengo claro que los datos del pasado deben formar parte del análisis, que ayuden a vislumbrar las posibles consecuencias de cualquier decisión de cambio del sistema, sin que limiten las opciones múltiples de futuro de una sociedad abierta y siempre en transformación.

El futuro es imaginación, innovación, cambio, que exige mentes progresistas. El pasado es certeza, realismo, estructuras establecidas, campo idóneo para razonamientos conservadores. Un ciudadano tanto como un técnico en cuestiones económicas, del mundo de hoy (y de siempre) debe mezclar ambas perspectivas: el realismo conservador y el cambio progresista. La cuestión es dar con las dosis correctas en cada caso. Y eso es un tema más ideológico que técnico.

Pero si estas consideraciones son válidas con carácter general, han cobrado más fuerza en entornos en transformación VICA creciente. Al menos desde los inicios de la crisis financiera en 2008-09, se ha intensificado la volatilidad o turbulencia de los cambios (tipo, velocidad, volumen y escala). Ha aumentado la incertidumbre sobre los acontecimientos condicionantes de toda predicción. Las interrelaciones económicas y geopolíticas se han vuelto más complejas y las consecuencias potenciales más ambiguas.

En estos entornos más volátiles, inciertos, complejos y ambiguos, ya no importa solo lo que es probable que ocurra, sino también lo que es posible, aunque su probabilidad sea baja según la experiencia hasta el momento en que se realiza la predicción (los cisnes negros).

Una predicción económica útil debe tener en cuenta que el camino hacia el futuro tiene senderos múltiples. Hay tendencias predominantes, pero también riesgos variados, con distinta probabilidad de ocurrencia y una incidencia diversa. Si el riesgo es acusado y el impacto alto, el predictor debe incorporarlo. Si el riesgo es reducido y su efecto pequeño, puede ser casi olvidado ¿Pero qué hacer con los $<$ cisnes negros $>$, desafíos poco probables pero de fuerte impacto? 
La discusión va más allá de la predicción y tiene sus antecedentes en los linderos de la filosofía, la dinámica de sistemas y el falsacionismo metodológico. Una teoría no puede ser aceptada como de validez general por el hecho de que se cumpla en diversas experiencias; siempre puede aparecer un extraño <cisne negro $>$, hasta ahora no avistado, que incumpla la ley propuesta. Un fenómeno residual y casi olvidado puede producir un caos en el sistema. La humanidad pudo vivir con la creencia errónea de que todos los cisnes eran blancos, hasta que en Australia se descubren cisnes negros a finales del siglo XVII.

En predicción la experiencia nos demuestra que los cisnes negros han jugado un papel esencial en los cambios radicales de tendencia. Los choques del petróleo, la Guerra del Golfo, los atentados de las Torres Gemelas, la quiebra de instituciones financieras en la Gran Crisis,..., han sido los acontecimientos clave de los fuertes quiebros de las economías del mundo y de los errores de muchas predicciones. Su impacto ha sido aún más amplio que en épocas anteriores por el refuerzo del <efecto mariposa $>$. El aleteo de las alas de una mariposa se puede, cada día más, sentir al otro lado del mundo. Lo que sucede en Irak, Siria, China o EEUU, afecta crecientemente a la evolución en la UE o en España.

Si un riesgo está escondido hasta que estalla repentinamente por un acto fortuito (p.ej. terrorismo) o una decisión política (un conflicto bélico), puede pensarse que estamos condenados a equivocarnos en los momentos más cruciales de la predicción. Eso es tan cierto como que el futuro no está escrito. Pero caben algunas actuaciones para paliar estas deficiencias de partida. La primera es tener en cuenta que existen pistas de un posible < cisne negro $>$ antes de que se produzca el estallido. La salida de Grecia del euro era muy poco probable, pero pudimos ir valorando su posible impacto. Como también una situación de guerra fría con Rusia, victorias del Estado Islámico o una eventual guerra cibernética. La imaginación sobre el futuro también ayuda a tomar decisiones (con todo su riesgo) aunque las predicciones sean más cualitativas que medidas en tasas o millones de euros.

Con riesgos de cisnes y efectos mariposa, pensar en cómo puede ser el futuro que nos espera sigue siendo una necesidad a cubrir. Eso sí, con la cautela imprescindible ante lo desconocido y la exigencia de buscar la información actualizada más relevante y revisar permanentemente nuestras apuestas de futuro.

Hoy día (y más aún cara al futuro) la predicción económica no puede limitarse a dar un valor del nivel o tasa de variación de las principales magnitudes de análisis, ni tampoco basta con añadir intervalos de confianza más o menos complejos. La clave está, sobre todo al ampliar el horizonte de predicción, en considerar las consecuencias de posibles rupturas en el entorno que pueden conducir a escenarios alternativos, en acentuar los rasgos prospectivos de una visión global, sistémica, dinámica y abierta, centrada en el análisis de distintos 
futuros alternativos en permanente revisión en un mundo en permanente transformación.

\section{REFERENCIAS BIBLIOGRÁFICAS}

AHIR, H. y LOUNGANI, P. (2014). There will be growth in the spring: How well do economists predict turning points?". VoxEU Organisation, 14 April.

FOX, S. y THORBECKE, E. (1968). The theory of Quantitative Economic Policy. North Holland Publishing Co.

HUERTA DE SOTO, J.(2004). Estudios de Economía Política. Madrid: Unión Editorial.

TETLOCK, P. y GARDNEr, D. (2015). Superforecasting: The art and science of prediction. Londres: Broad Books. 
